\title{
A deep learning-based cardio-vascular disease diagnosis system
}

\author{
Hamdan Bensenane, Djemai Aksa, Walid Fethallah Omari, Abdellatif Rahmoun \\ Department of Computer Science, Ecole Supérieure en Informatique (ESI-SBA), Sidi Bel-Abbes, Algeria
}

\section{Article Info}

Article history:

Received Aug 20, 2021

Revised Dec 8, 2021

Accepted Dec 17, 2021

\section{Keywords:}

Cardiac arrhythmia

Deep learning

ECG classification

Long-short term memory neural network

LSTM

ABSTRACT

Recently ehealth technologies are becoming an overwhelming aspect of public health services that provides seamless access to healthcare information. Machine learning tools associated with IoT technology play an important role in developing such health technologies. This paper proposes a decision support system-based system (DSS) to make diagnosis of cardiovascular diseases. It uses deep learning approaches that classify electrocardiogram (ECG) signals. Thus, a two-stage long-short term memory (LSTM) based neural network architecture, along with an adequate preprocessing of the ECG signals is designed as a diagnosis-aided system for cardiac arrhythmia detection based on an ECG signal analysis. This deep learning based cardio-vascular disease diagnosis system (namely 'DLCVD') is built to meet higher performance requirements in terms of accuracy, specificity, and sensitivity. This must also be capable of an online real-time classification. Experimental results using the Massachusetts Institute of Technology-Beth Israel Hospital (MIT-BIH) arrhythmia database show that DLCVD led to outstanding performance.
\end{abstract}

This is an open access article under the CC BY-SA license.

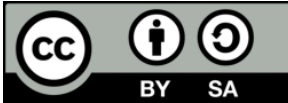

\section{Corresponding Author:}

Hamdan Bensenane

Department of Computer Science, Higher School in Computer Science (ESI-SBA)

22000 Sidi Bel-Abbes, Algeria

Email: h.bensenane@esi-sba.dz

\section{INTRODUCTION}

A recent study of the World Health Organization (WHO) revealed that cardiovascular diseases are considered the leading cause of death in the world, with more than 18 million deaths per year. Consequently, several projects have been carried out to implement computer-assisted diagnostic systems. However, few works have led to applicable results in real-life situations. Therefore, several research works have been carried out to design a variety of diagnostic models for this type of disease. As shown in [1], we can design a diagnostic model of cardiac arrhythmia. In recent years, many neural network models have demonstrated remarkable performance in predicting data and solving classification problems. Deep learning methods such as convolutional neural networks (CNNs) and long-term memory networks (LSTMs) have an important place in smart health applications such as disease classification. Therefore, our goal in this work is to present a new classification approach for 17 arrhythmia types and the resolution of data set imbalance problems. This approach uses LSTM classification of ECG signals.

As published in recent studies, arrhythmia detection research presents several challenges [2]. In this section, we will mention the essential elements that help to develop a decision support system for disease diagnosis. With respect to beats and rhythm, there are distinct approaches in the literature based on the classification of a single heartbeat (QRS complex), or sometimes a set of subsequent heartbeats. However, due to the incorrect classification of cardiac disorders based on a single cardiac cycle [3], the examination of a longer ECG duration resulted in better performance in identifying arrhythmic diseases as in [4], [5]. 
In summary, the search strategy for ECG signal analysis presented in the review articles [2], [6], [7] includes the following steps:

- Obtain a database of public and/or private ECG signals such as (MIT-BIH, NST, and QT).

- Preprocessing and signal normalization.

- Heartbeat (QRS complex) detection and/or ECG signal segmentation.

- Extraction, transformation and selection of ECG features.

- Classification of heartbeats or heart rhythm (cardiac anomaly recognition).

- Results evaluation.

Other considerations concern inter- and intra-patient paradoxes. Validation schemes provide a measure of the performance and generalization ability of the computational model [2], [8].

- Inta-patient validation schemes: Test and train data sets are selected from the same patient's signals.

- Inter-patient validation schemes: Test and train data sets are selected from different patient's signals [9].

Several research papers deal with ECG signals to design systems to aid in the diagnosis of cardiovascular diseases. Plawiak [5], tested probabilistic neural network (PNN), radial basis function network (RBFNN), k-nearest neighbor (KNN), and support-vector machines (SVM) with genetic algorithm-based optimization (GA). In [10], [11], authors used a Gaussian mixture model of Karhunen-Loève transform. In [5], the authors combined the genetic algorithm with SVM. Methods based on the application of a onedimensional CNN were used in [12], [13] and gave results above 90\% accuracy [13]. In [14], authors proposed two novel methods for multiclass ECG arrhythmias classification based on principal components analysis (PCA), fuzzy support vector machine and unbalanced clustering.

The methods for classifying cardiac arrhythmias use the same MIT-BIH database composed of 17 arrhythmia categories. In the second approach, the classification of the heart rhythm (QRS complex) is built on the classification of 2 to 7 classes [15]. In other approach the use of discrete wavelet transformation (DWT) preprocessing with MLP, RBFNN, PNN to detect normal and abnormal beats is explained in [16]-[18]. Similarly in [19], [20], the authors used LSTM and RNN-LSTM with performance equal to $99.99 \%$. In [21]-[23], the authors used normalization with CNN and DWT combined with fast R-CNN. In terms of accuracy, in [24], the authors tested SVM and BPNN with genetic optimization and without. For the GA-BPNN approach, the authors obtained an accuracy of $97.87 \%$. Olanrewaju et al. [25] proposed a method to accurately predict heart disease using continuous wavelet transform (CWT) and deep neural deep neural networks.

The proposed methods for the classification of cardiac arrhythmias are important in terms of results and studies performed. The problem is that most of these methods remained theoretical studies and they were not used for real diagnostic systems. The first assumption we made is that the ECG signal alone is enough to make a fairly acceptable diagnosis of several arrhythmias. The second assumption is that the LSTM should be capable of attaining good results since it is designed to work with different kinds of time-series data like ECG signals.

\section{METHOD}

\subsection{Data set}

The database used in our work is composed of 1000 randomly selected 10-second fragments of ECG signals obtained from the MIT-BIH arrhythmia database [26]. The sequences are recorded at a sampling rate of $360 \mathrm{~Hz}$. Therefore, each fragment contains 3600 samples representing 10 seconds of recordings with a single ECG lead position (MLII). The database is composed of records belonging to 45 patients, 19 females (age range 23-89 years) and 26 males (age range 32-89 years). This database contains 17 distinct classes between normal sinus rhythm (NSR) and 16 types of arrhythmia as shown in Table 1. To test our approach, we used two sets, a training set and a validation set with a 90-10 ratio. The data set is random, this way of organizing the database will give an equal chance to each sample to be present during the learning and validation step.

\subsection{Preprocessing}

First, we normalized the data by reducing the gain and then applying constant component reduction and re-scaling the data to the range of $[-1,1]$. Thereafter, the Pan-Tompkins algorithm [27] is applied to each ECG fragment in order to detect the R-peaks. This helps create windows (frames) around the R-peaks. Various widths of windows were tested and the width of 300 points gave the best results. In each frame, the R-peak is set as a center.

Constant component reduction, as in (1), for an ECG signal $x$,

$$
\mu=\frac{1}{n} \sum_{i=1}^{n} x(i)
$$


- $\mathrm{n}$ : number of signal samples (in this case $=3600$ )

- $\mathrm{i}$ : index of consecutive signal samples

Rescalling, as in (2), for a signal fragment $x$,

$$
\mathrm{x}=2 \cdot \frac{\mathrm{x}(\mathrm{i}) \cdot \min (\mathrm{x})}{\max (\mathrm{x})-\min (\mathrm{x})}
$$

- i : index of consecutive signal samples

- $\min (x)$ : minimum signal amplitude value

- $\max (x)$ : maximum signal amplitude value

Table 1. Dataset decomposition

\begin{tabular}{clcccc}
\hline No & \multicolumn{1}{c}{ Class } & $\begin{array}{c}\text { Nber of } \\
\text { samples }\end{array}$ & $\begin{array}{c}\text { Nber of } \\
\text { patients }\end{array}$ & $\begin{array}{c}\text { Training } \\
\text { set }\end{array}$ & $\begin{array}{c}\text { Validation } \\
\text { set }\end{array}$ \\
\hline 1 & Normal sinus rhythm & 283 & 23 & 255 & 28 \\
2 & Ventricular tachycardia & 10 & 3 & 9 & 1 \\
3 & Idioventricular rhythm & 10 & 1 & 9 & 1 \\
4 & Ventricular flutter & 10 & 1 & 9 & 1 \\
5 & Fusion of ventricular and normal beat & 11 & 3 & 10 & 1 \\
6 & Left bundle branch block beat & 103 & 3 & 93 & 10 \\
7 & Right bundle branch block beat & 62 & 3 & 56 & 6 \\
8 & Second-degree heart block & 10 & 1 & 9 & 1 \\
9 & Pacemaker rhythm & 45 & 2 & 40 & 5 \\
10 & Atrial premature beat & 66 & 9 & 59 & 7 \\
11 & Atrial flutter & 20 & 3 & 18 & 2 \\
12 & Atrial fibrillation & 135 & 6 & 121 & 14 \\
13 & Supraventricular tachyarrhythmia & 13 & 4 & 12 & 1 \\
14 & Pre-excitation (WPW) & 21 & 1 & 19 & 2 \\
15 & Premature ventricular contraction & 133 & 14 & 120 & 13 \\
16 & Ventricular bigeminy & 55 & 7 & 49 & 6 \\
17 & Ventricular trigeminy & 13 & 4 & 12 & 1 \\
\hline
\end{tabular}

Figure 1 describes each step of the preprocessing stage of an ECG signal and normalization before being fed to the classification model. Figure 1(a) illustrates an ECG fragment before preprocessing, Figure 1(b) demonstrates a normalized ECG fragment, Figure 1(c) shows the R-peaks detected in the ECG signal with the Pan-Tompkins algorithm, and Figure 1(d) shows the result of the ECG signal segmentation into windows. Usually LSTM neural networks perform good performance when it comes to processing sequential and temporal data, making them perfect for ECG signal classification tasks.

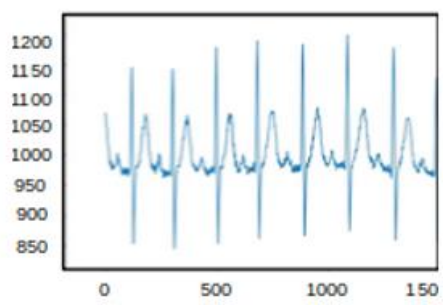

(a)

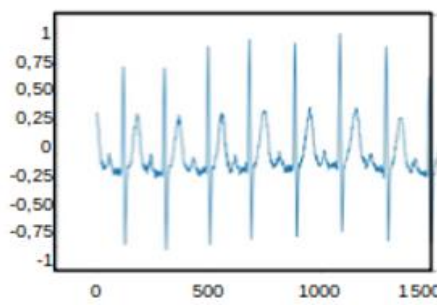

(b)

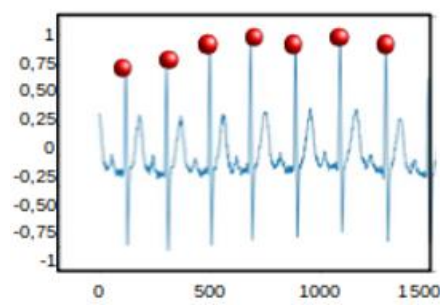

(c)
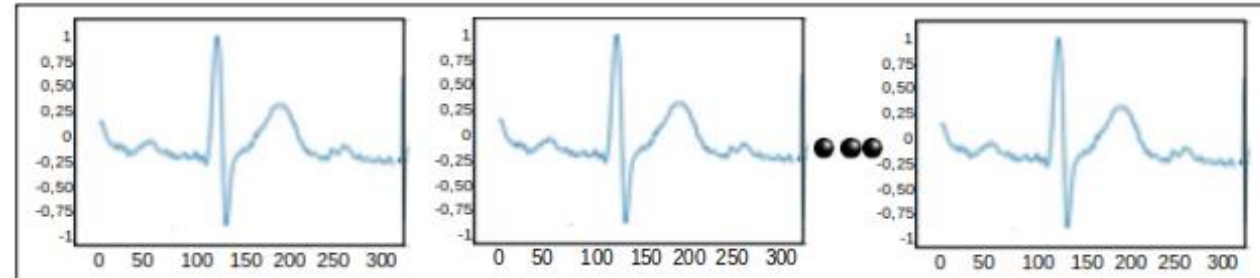

(d)

Figure 1. Preprocessing and normalization of an ECG sample, (a) ECG fragment before preprocessing, (b) Normalized ECG signal, (c) R-peak detection with Pan-Tompkins algorithm, and (d) Signal segmentation to windows 
Figure 2 illustrates a general architecture where:

$-w$ : number of layers.

- $h_{0}, c_{0}:$ initial hidden and cell state.

- $h_{n}, c_{n}$ : last hidden and cell state.

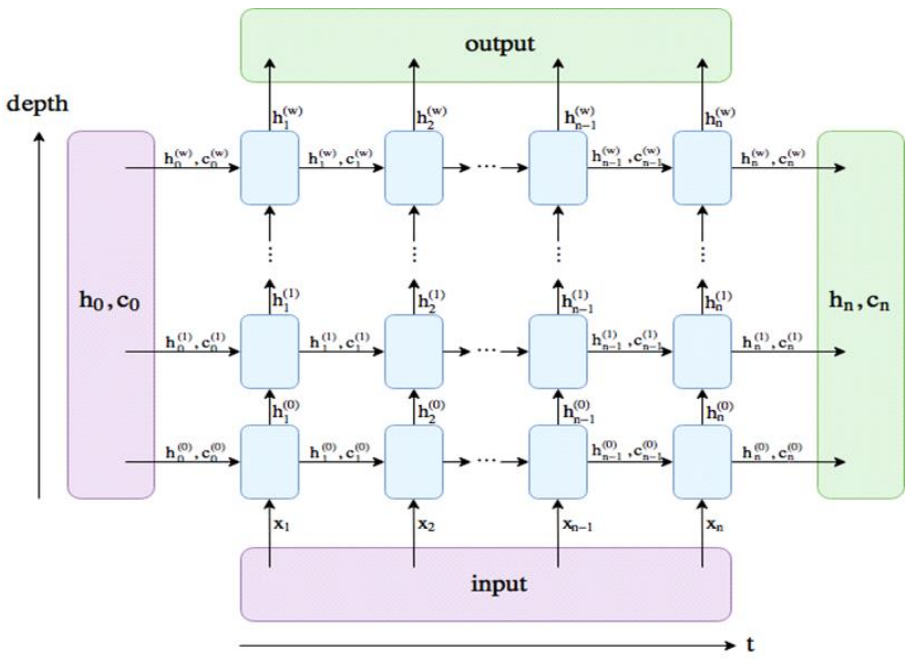

Figure 2. An overview of LSTM architecture [24]

\section{3. Arrhythmia classification model design}

The proposed model, named deep learning based cardio-vascular disease diagnosis system (DLCVD), consists of two LSTM layers, each consisting of 128 and 64 hidden nodes respectively. This particular architecture, separating the LSTM layers and stacking them instead of using one LSTM layer with two (or more) hidden layers gave us more control over the network and allowed us to do better fine tuning, while using less computational time. The stacked LSTM layers are followed by a dropout of 0.4 probability and two dense layers of (64-64) and (64-17) as input-output size respectively. Table 2 shows the network layers and their parameters. The network architecture is described in Figure 3. The training was carried out with a batch size of one, meaning that the weights of the network are updated after each element of the dataset is passed through the network.

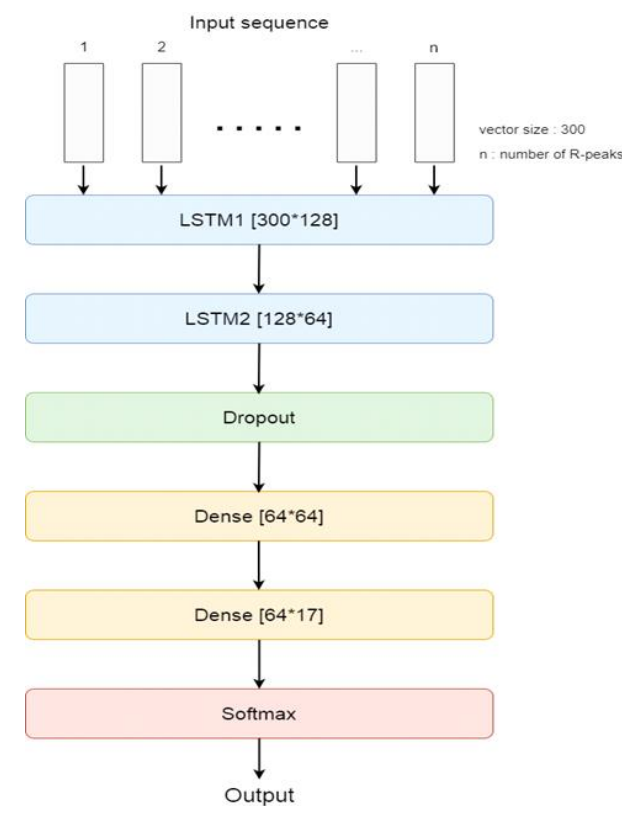

Figure 3. Block diagram of the $D L C V D$ architecture 
Table 2. Network parameters

\begin{tabular}{ccl}
\hline No & Layer & \multicolumn{1}{c}{ Parameters } \\
\hline 1 & LSTM & Number of layers: 1 Hidden nodes: 128 Input size: 300 \\
2 & LSTM & Number of layers: 1 Hidden nodes: 64 Input size: 128 \\
3 & Dropout & Drop probability: 0.4 \\
4 & Dense & Input size: 64 output size: 64 \\
5 & Dense & Input size: 64 output size: 17 \\
& Training $\sim 10$ seconds & per epoch \\
\hline
\end{tabular}

\section{RESULTS AND DISCUSSION}

Due to the small size of the dataset and the imbalance between various classes, augmenting data is necessary. We used stratified 10 folds cross validation in order to have a better, realistic and objective estimate of performance of the model. Hence, 10 training subsets of 900 samples each and 10 validation subsets of 100 samples each complementing the training subsets were created, with a ratio of 9 to 1 (training/validation sets). The number of samples in training and validation sets for each class is described in Table 1. The training phase took 50 epochs for each fold and the validation loss reached its lowest rate somewhere around the 40th and 50th epoch. The validation loss of each epoch in each fold was summed up and the mean of loss in each epoch of the 10 folds is represented in Figure 4.

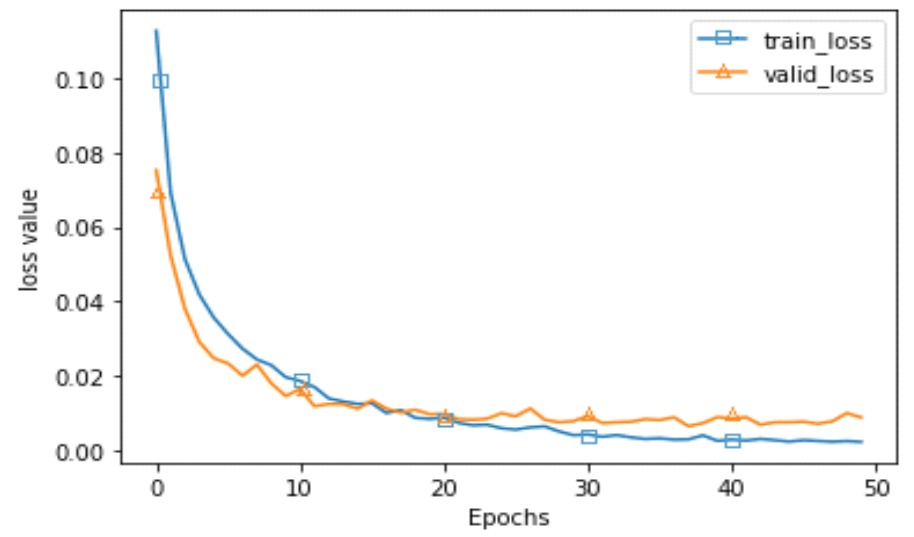

Figure 4. Training and validation loss

As a measure of performance, we used multiple criteria namely accuracy, sensitivity (or recall) and specificity. Accuracy (ACC), as in (3), gives a general view on how samples are well classified. The LSTM model obtained $93 \%$ accuracy on the best fold and an average accuracy of $89.30 \%$.

$$
A C C=\sum_{i=1}^{n} \frac{T P+T N}{T P+F P+T N+F N} \frac{100 \%}{N}
$$

Sensitivity (recall), as in (4), depicts the actual proportion of positive samples correctly predicted, and the model was capable to achieve $95.31 \%$ sensitivity with an overall average sensitivity of $82.85 \%$.

$$
S E N=\sum_{i=1}^{n} \frac{T P}{T P+F P+T N+F N} \cdot \frac{100 \%}{N}
$$

Specificity, as in (5), reviews the proportion of negatives predicted correctly, and reached $99.46 \%$ with an average of $99.21 \%$.

$$
S P E=\sum_{i=1}^{n} \frac{T N}{T P+F P+T N+F N} \cdot \frac{100 \%}{N}
$$

The confusion matrix, illustrated in Figure 5, shows the sum of each one of the 10 folds. The proposed model successfully classified 893 out of 1000 samples (sum of all 10 folds), and the major confusion occured between classes 10 "atrial premature beat" and 15 "premature ventricular contraction" being confused with class 1 "normal sinus rhythm". This is due to some similarities between the classes, also the imbalance in the dataset where class 1 is dominating the dataset as seen in Table 1. Other minor 
confusion occurred between some of the other classes, which are totally predictable keeping in mind that the dataset contained only 1,000 samples. Figure 6 represents the average metrics of the 10 folds for each class. The model attained a near perfect specificity making it capable of ruling out the false positives with $99.21 \%$ success rate. The lowest metrics were observed for class 13 "supraventricular tachyarrhythmia" followed by class 5, "fusion of ventricular and normal beat". Both classes only had 13 and 11 samples in the dataset respectively, of which, only one sampling was used for validation in each fold. The implementation and evaluation were conducted on Core i7-4790 CPU-8GB RAM. As we advocated the good performance produced by applying our LSTM model on MIT-BIH dataset into classifying 17 types of arrhythmia, we ought to give an overview of how much our method fares compared to other methods as highlighted in Table 3. Granted that we applied the same preprocessing stage, and that other methods adopted optimization algorithms to fine tune the parameters and the feature selection process, our method that uses a 2-stage LSTM produced good results, as compared to the 1D-CNN in [12]. Furthermore, unlike the methods proposed in [5], [6], we fall short when it comes to accuracy. Although accuracy can be deceiving especially when dealing with imbalanced dataset; it suffices to recognize the major classes correctly to get a higher accuracy. However, the proposed method have achieved higher sensitivity and specificity $(95.31 \%$ and $99.46 \%$ respectively) which are more reliable as assessment scores in medical diagnosis systems performance.

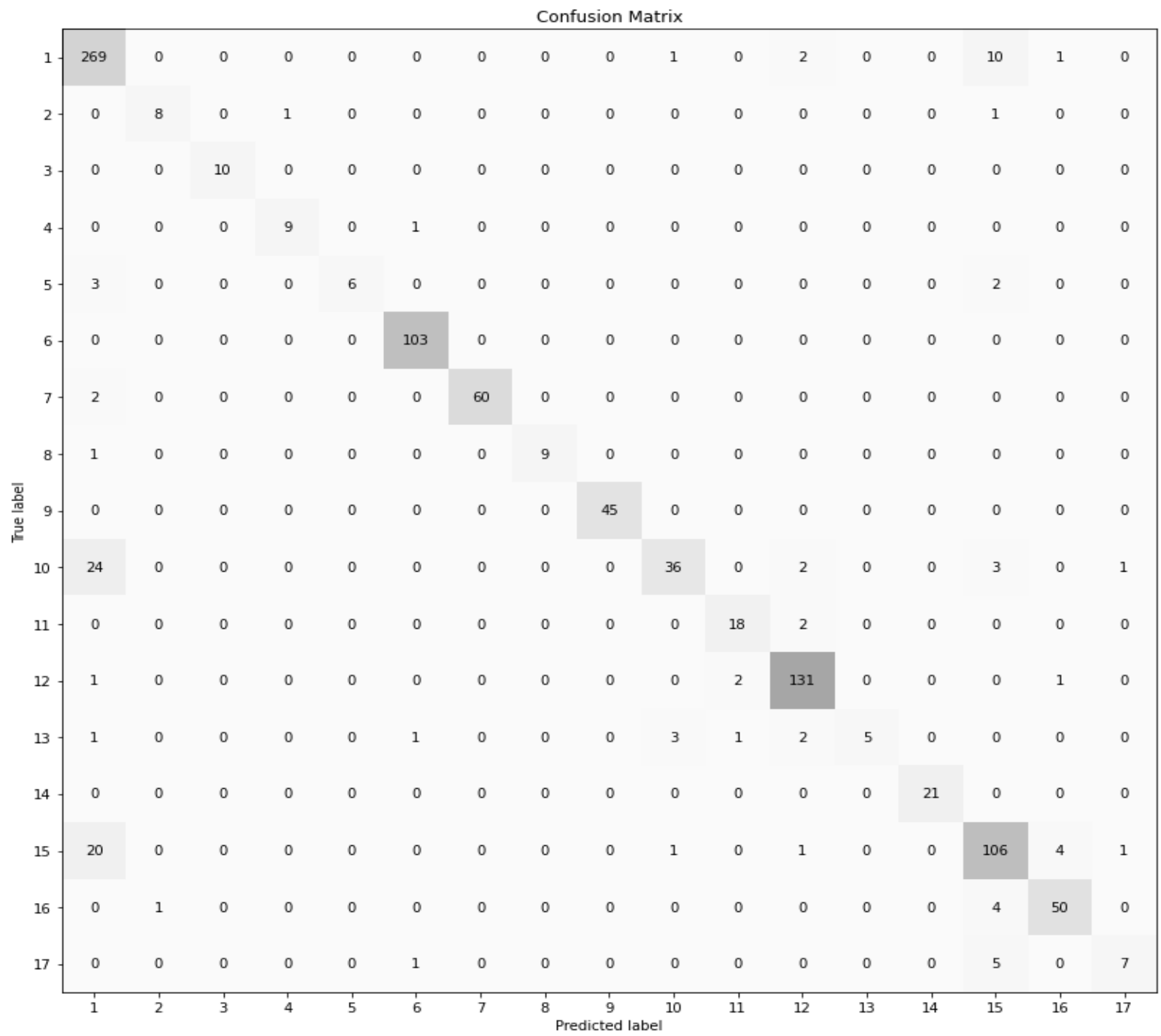

Figure 5. Confusion matrix summing the 10 folds 


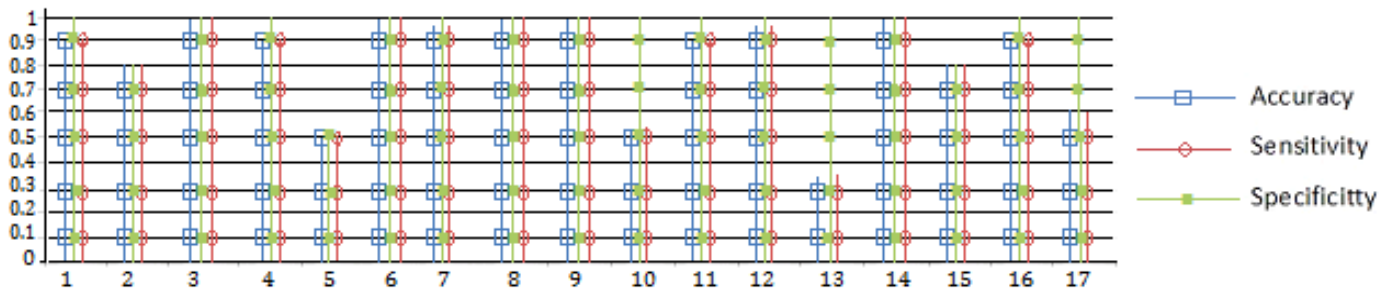

Figure 6. An overview of classes the averaged metrics between all the 10-fold cross-validation processes

Table 3. A comparison of the results of the methods applied on the same database MIT-BIH arrhythmia

\begin{tabular}{ccccccc}
\hline Paper & No of classes & Preprocessing & Method & Accuracy & Sensitivity & Specificity \\
\hline & & & GA + KNN & $98.52 \%$ & $87.45 \%$ & $99.22 \%$ \\
Plawiak [4] & \multirow{2}{*}{17} & Normalization & GA + PNN & $98.62 \%$ & $88.25 \%$ & $99.27 \%$ \\
& & & GA+RBFN GA + SVM & $98.63 \%$ & $88.30 \%$ & $99.27 \%$ \\
& & & $98.75 \%$ & $89.35 \%$ & $99.34 \%$ \\
Mohebbanaaz et al. [12] & \multirow{2}{*}{17} & Normalization & 1D-CNN & $91.33 \%$ & $83.91 \%$ & $99.41 \%$ \\
Plawiak [5] & 17 & Normalization & GA+GECS-SVM & $98.99 \%$ & $91.10 \%$ & $99.46 \%$ \\
The proposed method & \multirow{2}{*}{17} & Normalization & LSTM: Average & $89.3 \%$ & $82.85 \%$ & $99.21 \%$ \\
$(D L C V D)$ & & & LSTM: Best results & $93 \%$ & $95.31 \%$ & $99.46 \%$ \\
\hline
\end{tabular}

Other aspects that have an important part in this research are the effects of preprocessing stages with its various techniques on the outcome of the interpretation models. In addition to the paradigms adopted to opt such as a single QRS complex or longer duration ECG analysis along with its implication as demands rises for diagnostic systems a certain level of abstraction is needed for the domain experts as it is interesting to diagnose diseases and not just general pointers as heartbeat annotations. Another part, which is one of the important things to address is the assessments schemes, as mentioned earlier in section 2.1 a generalized model usually is trained on data from diffrent patients (inter-patient evaluation), however the limited source of data may prevent the application of such approach. While the methods varies to get more insight on this classification problem, the choice in our study can be justified by the reputation LSTMs have in dealing with time-series data and the results we got in this experiment.

\section{CONCLUSION}

This paper presents DLCVD, a two-stage deep learning LSTM neural network to classify arrhythmias ("normal sinus rhythm", "pacemaker rhythm" and 15 rhythm disorders) using fragments of 10 seconds long of ECG signal. This paper demonstrates that a proper pre-processing along with a two-stage LSTM neural network, enhance accuracy in classifying cardio-vascular diseases using ECG signals. Despite the limited and imbalanced dataset, our proposed model achieved good performance in terms of accuracy, sensitivity and specificity respectively. It is also shown through experiments, and compared to well-known machine learning classification techniques in the current literature, that DLCVD reveals good classification performance. The proposed model is resource friendly and capable of real time classification. It is also universal and offers high accuracy, sensitivity and specificity, simple to use and can easily be adapted to different use cases and scenarios, and requires low computational resources. Future work will emphasize on using this model to classify other physiological signals (such as EEG and EMG), Increasing performance using a larger ECG dataset size, using a larger width of ECG frames, and providing more samples within frames. This would certainly require more computational resources.

\section{ACKNOWLEDGEMENTS}

We would like to express our appreciation to all those who have supported this research work at the School of Computer Science (ESI-SBA, Algeria); as well as research team of the Computational Intelligence and Soft Computing (CISCo), LabRI, laboratory at 'Ecole Supérieure en Informatique', Sidi Bel-Abbes, Algeria.

\section{REFERENCES}

[1] J. Verjans and T. Leiner, “Artificial intelligence for the general cardiologist,” Netherlands Heart Journal, vol. 27, no. 9, pp. 389391, 2019, doi: 10.1007/ s12471-019-01327-7.

[2] E. J. d.S. Luz, W. R. Schwartz, G. C.-Cháveza, and D. Menotti, "ECG-based heartbeat classification for arrhythmia detection: A survey," Computer Methods and Programs in Biomedicine, vol. 127, pp. 144-164, 2016, doi: 10.1016/j.cmpb.2015.12.008. 
[3] K. Padmavathi and K. Sri Ramakrishna, "Classification of ECG signal during atrial fibrillation using autoregressive modeling," Procedia Computer Science, vol. 46, pp. 53-59, 2015, doi: 10.1016/j.procs.2015.01.053.

[4] P. Pławiak, "Novel genetic ensembles of classifiers applied to myocardium dysfunction recognition based on ECG signals," Swarm and Evolutionary Computation, vol. 39, pp. 192-208, 2018, doi: 10.1016/j.swevo.2017.09.002.

[5] P. Plawiak, "Novel methodology of cardiac health recognition based on ECG signals and evolutionary-neural system," Expert Systems with Applications, vol. 92, pp. 334-349, 2018, doi: 10.1016/j.eswa.2017.09.022.

[6] E. G. D. S. Luz, T. M. Nunes, V. H. C. de Albuquerque, J. P. Papa, and D. Menotti, "ECG arrhythmia classification based on optimum-path forest," Expert Systems with Applications, vol. 40, no. 9, pp. 3561-3573, 2013, doi: 10.1016/j.eswa.2012.12.063.

[7] E. Kańtoch, M. Smoleń, P. Augustyniak, and P. Kowalski, "Wireless body area network system based on ECG and accelerometer pattern," Proc. Computing in Cardiology, 2011, pp. 245-248.

[8] R. G. Afkhami, G. Azarnia, and M. A. Tinati, "Cardiac arrhythmia classification using statistical and mixture modeling features of ECG signals," Pattern Recognition Letters, vol. 70, pp. 45-51, 2016, doi: 10.1016/j.patrec.2015.11.018.

[9] P. D. Chazal, M. O.'Dwyer, and R. B. Reilly, "Automatic classification of heartbeats using ECG morphology and heartbeat interval features," IEEE Transactions on Biomedical Engineering, vol. 51, no. 7, pp. 1196-1206, 2004, doi: 10.1109/TBME.2004.827359.

[10] P. Crippa, A. Curzi, L. Falaschetti, and C.Turchetti, "Multi-class ECG beat classification based on a gaussian mixture model of karhunen-loève transform," International Journal of Simulation: Systems, Science \& Technology, vol. 16, no. 1, pp. 1-10, 2015, doi: 10.5013/IJSSST.a.16.01.02.

[11] G. Biagetti, P. Crippa, A. Curzi, S. Orcioni, and C. Turchetti, "A multi-class ECG beat classifier based on the Truncated KLT representation," Proc. European Modelling Symposium, 2014, pp. 93-98, doi: 10.1109/EMS.2014.31.

[12] Mohebbanaaz, Y. P. Sai, and L. V. R. Kumari, "Detection of cardiac arrhythmia using deep CNN and optimized SVM," Indonesian Journal of Electrical Engineering and Computer Science (IJEECS), vol. 24, no. 1, pp. 217-225, 2021, doi: 10.11591/ijeecs.v24.i1.pp217-225.

[13] Ö. Yıldırım, P. Pławiak, R.-S. Tan, and U. R. Acharya, “Arrhythmia detection using deep convolutional neural network with long duration ECG signals," Computers in Biology and Medicine, vol. 102, pp. 411-420, 2018, doi: 10.1016/j.compbiomed.2018.09.009.

[14] M. Nait-Hamoud and A. Moussaoui, "Two novel methods for multiclass ECG arrhythmias classification based on PCA, fuzzy support vector machine and unbalanced clustering," Proc. International Conference on Machine and Web Intelligence, 2010, pp. 140-145, doi: 10.1109/ICMWI.2010.5647931.

[15] H. Lassoued, R. Ketata, and S. Yacoub, "ECG decision support system based on feedforward neural networks," International Journal on Smart Sensing and Intelligent Systems, vol. 11, no. 1, pp. 1-15, 2018, doi: 10.21307/ ijssis-2018-029.

[16] M. Kachuee, S. Fazeli, and M. Sarrafzadeh, "ECG heartbeat classification: a deep transferable representation," Proc. IEEE International Conference on Healthcare Informatics, 2018, pp. 443-444, doi: 10.1109/ICHI.2018.00092.

[17] M. Thomas, M. Kr. Das, and S. Ari, "Automatic ECG arrhythmia classification using dual tree complex wavelet-based features," AEU-International Journal of Electronics and Communications, vol. 69, no. 4, pp. 715-721, 2015, doi: 10.1016/j.aeue.2014.12.013.

[18] S. L. Oh, E. Y. K. Ng, R. S. Tan, and U. R. Acharya, "Automated diagnosis of arrhythmia using combination of CNN and LSTM techniques with variable length heart beats," Computers in Biology and Medecine, vol. 102, pp. 278-287, 2018, doi: 10.1016/j.compbiomed.2018.06.002.

[19] S. Singh, S. K. Pandey, U. Pawar, and R. R. Janghel, "Classification of ECG arrhythmia using recurrent neural networks," Procedia Computer Science, vol. 132, pp. 1290-1297, 2018, doi: 10.1016/j.procs.2018.05.045.

[20] T. Li and M. Zhou, "ECG classification using wavelet packet entropy and random forests," Entropy, vol. 18, no. 8, pp. 285, 2016, doi: 10.3390/e180802.

[21] Y. Ji, S. Zhang, and W. Xiao, "Electrocardiogram classification based on faster regions with convolutional neural network," Sensors, vol. 19, no. 11, pp. 2558, 2019, doi: 10.3390/s19112558.

[22] A. Sellami and H. Hwang, "A robust deep convolutional neural network with batch weighted loss for heartbeat classification," Expert Systems with Applications, vol. 122, pp. 75-84, 2019, doi: 10.1016/j.eswa.2018.12.037.

[23] P. S. Lakshmi and V. L. Raju, "ECG De-noising using hybrid linearization method," Indonesian Journal of Electrical Engineering and Computer Science (IEECS), vol. 15, no. 3, pp. 504-508, 2015, doi: 10.11591/telkomnika.v15i3.8427.

[24] H. Li, D. Yuan, X. Ma, D. Cui, and L. Cao, "Genetic algorithm for the optimization of features and neural networks in ECG signals classification,” Scientific Reports, vol. 7, no. 1, pp. 1-12, 2017, doi: 10.1038/srep41011.

[25] R. F. Olanrewaju, S. N. Ibrahim, A. L. Asnawi, and H. Altaf, "Classification of ECG signals for detection of arrhythmia and congestive heart failure based on continuous wavelet transform and deep neural networks," Indonesian Journal of Electrical Engineering and Computer Science (IJEECS), vol. 12, no. 1, pp. 1520-1528, 2021, doi: 10.11591/ijeecs.v22.i3.pp1520-1528.

[26] G. B. Moody and R. G. Mark, "The impact of the MIT-BIH arrhythmia database," IEEE Engineering in Medicine and Biology Magazine, vol. 20, no. 3, pp. 45-50, 2001, doi: 10.1109/51.932724.

[27] J. Pan and W. J. Tompkins, "A real-time QRS detection algorithm," IEEE Transactions on Biomedical Engineering BME, vol. 32, no. 3, pp. 230-236, 1985, doi: 10.1109/TBME.1985.325532.

\section{BIOGRAPHIES OF AUTHORS}

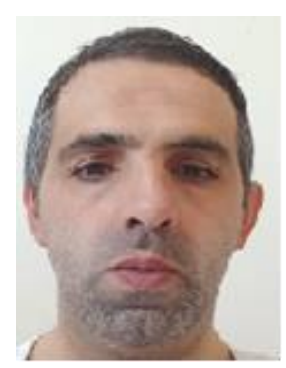

Hamdan Bensenane (D) 8d SC P in Computer Science, and Associate Professor at ESISBA School of; The Higher Computer Science, Sidi Bel Abbes, Algeria. He has been teaching Computer Science Materials, and very interested in research in Machine Learning, IOT, and Embedded Systems for more than six years. He can be contacted at email: h.bensenane@esi-sba.dz. 

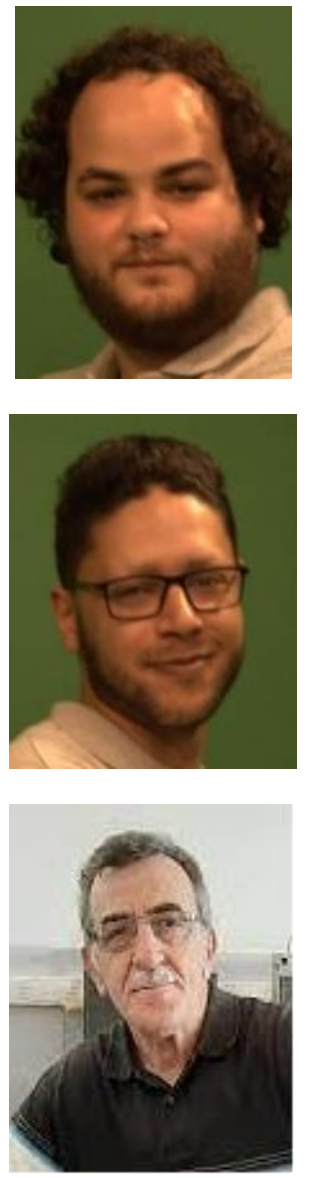

Djemai Aksa (iD I8 SC P student in Computer Science. He has been intensively involved in the area of Embedded Systems, IOT and Machine Learning. He can be contacted at email: d.aksa@esi-sba.dz.

Walid Fethallah Omari (D) 81 SC P student in Computer Science. He has been intensively involved in the area of Embedded Systems, IOT and Machine Learning. He can be contacted at email: f.omari@esi-sba.dz.

Abdellatif Rahmoun, Ph.D (D) SC P in Computer Science, and Full Professor at ESISBA. The Higher School Of Computer Science, Sidi Bel Abbes, Algeria. He has been teachning Computer Science Materials, and very interested in research in Machine Learning, IOT, and Embedded Systems for more than twenty years. He can be contacted at email: a.rahmoun@esi-sba.dz. 